\title{
Retroperitoneal Gas Gangrene Complicating Elective Inguinal Hernia Repair
}

\author{
A Privitera, DP Edwards, C Cunningham
}

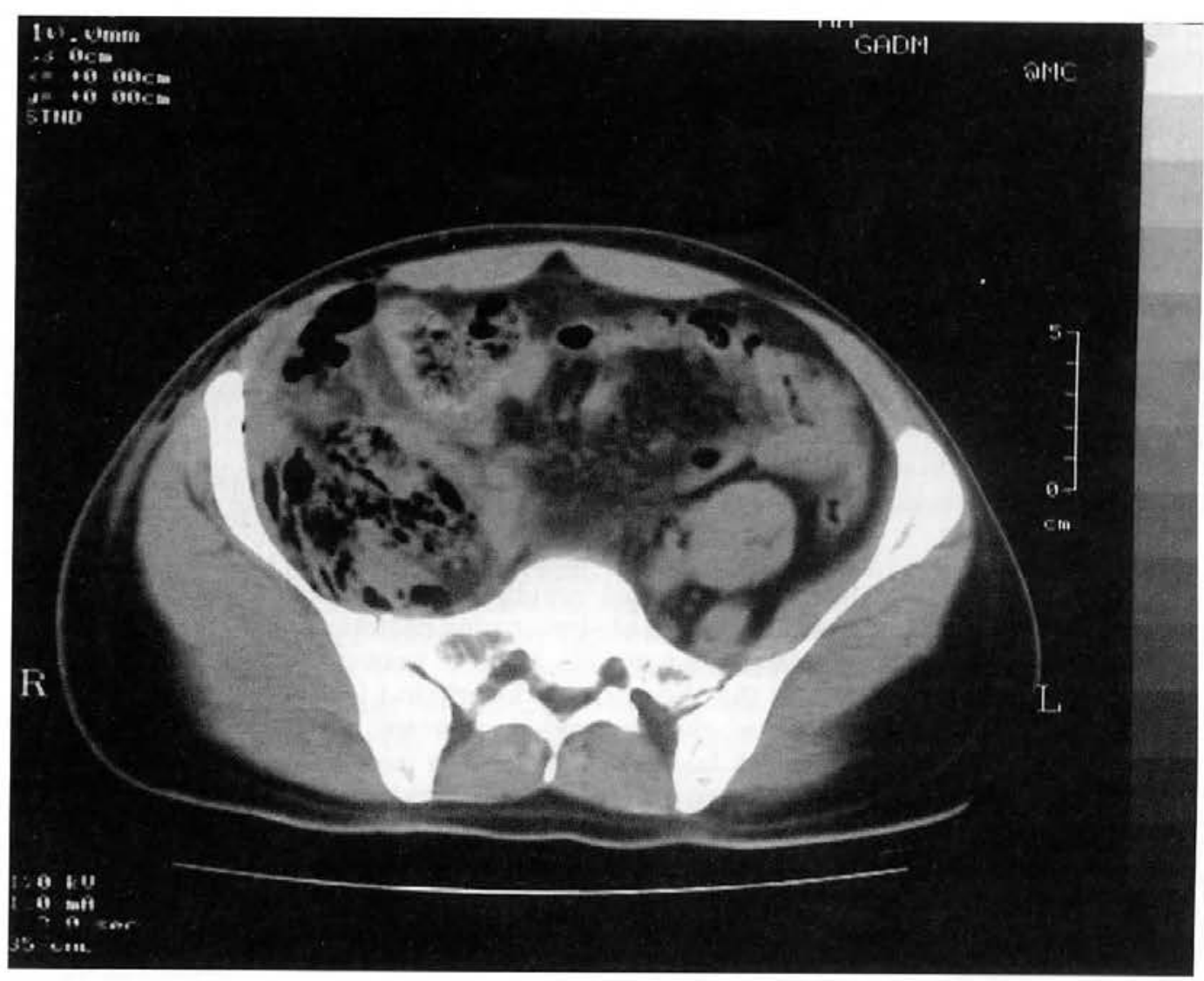

Dr A Privitera MD

Senior House Officer in Surgery

Maj D P Edwards

ChM FRCSEd

Specialist Registrar in

Surgery

Mr C Cunningham

MD FRCSEd

Consultant Surgeon

Department of

Colorectal Surgery,

Level 2, John Radcliffe

Hospital,

Headley Way,

Headington,

Oxford, OX3 9DU

\section{ABSTRACT}

Gas gangrene is a well recognised complication of contaminated military wounds. A case of Clostridial myonecrosis following elective inguinal hernia repair is described. The pathology, clinical features and management of this life-threatening condition are reviewed.

\section{Introduction}

Necrotising soft-tissue infections are uncommon life-threatening conditions associated with a high morbidity and mortality (1). Clostridial myonecrosis is a distinct necrotising infection of the skeletal muscle with dramatic pathological features and fulminant clinical course. The organisms responsible are large gram-positive anaerobic rods that possess a central sub-terminal spore. Six species cause gas gangrene in man. C. perfringens is the most commonly recovered and next in frequency are C. novyi and C. septicum (2). We report the case of a patient with retroperitoneal myonecrosis due to C. perfringens infection following an elective inguinal hernia repair.
Fig 1. CT Scan of lower abdomen showing gas and oedema in planes of right retroperitoneal muscles.

\section{Case History}

A 24-year-old man was transferred to intensive care facilities from another hospital with a 24 hour history of severe right iliac fossa pain radiating to the loin and leg, two days after an elective right inguinal hernia Lichtenstein repair. He was haemodynamically unstable exhibiting clinical signs and haematological parameters of severe sepsis and multi-system failure. His abdomen was tender, particularly in the right iliac fossa, with a discrete region of crepitus at the lateral margin of an otherwise unremarkable inguinal wound. A CT scan performed prior to transfer demonstrated extensive gas formation and muscle destruction in the right retroperitoneal compartment (Figure 1). Surgical debridement was immediately undertaken following aggressive fluid resuscitation, with inotropic support, ventilation and antibiotics (clindamycin and cefuroxime).

The abdomen was opened through a midline laparotomy incision. There was no 
evidence of bowel injury as a consequence of hernia repair or other gastrointestinal pathology. The right inguinal hernia wound was excised en bloc with the prosthetic mesh and the incision extended from the right flank to the costal margin. A tract of dead muscle led from the site of repair to the retroperitoneal compartment with extensive destruction and liquefaction of psoas, quadratus lumborum and iliacus muscles. These were excised, skeletalising the neurovascular structures, ureter and vertebral muscle attachments. The abdomen was closed and the retroperitoneal wound packed. Minimal further debridement was performed when packs were changed 24 hours later. After 8 days in intensive care the patient was transferred to the ward and was discharged 10 days later. Despite the extensive debridement he was independently ambulant by this stage. Histological examination of the muscle revealed myonecrosis and acute myositis, cultures of reported profuse growth of C. perfringens.

\section{Discussion}

Necrotising soft tissue infection is characterized by rapidly progressing inflammation and life-threatening necrosis of skin, fascia and muscle. It most frequently involves the abdomen, perineum and lower extremities. Surgery and trauma are often implicated, but spontaneous cases have also been reported $(3,4)$. Patients at higher risk include the elderly, the immunocompromised, the malnourished, the obese, diabetics and intravenous drug abusers $(3,5)$.

Infections can be classified by causative organisms: polymicrobial or necrotising fasciitis type $\mathrm{I}$, and monomicrobial including necrotising fasciitis type II (due to group A streptococcus) and gas gangrene (clostridial myonecrosis)(6). Nichols and Smith suggested that necrotising infections within 48 hours of surgery are likely to be monomicrobial (7).

Bacterial exotoxins such as those produced by C. perfringens and Streptococcus pyogenes, cause tissue necrosis. C. perfringens produces 12 toxins the most important of which the alfa-toxin, a phospholipase which degrades lecithin (a major component of cell membranes) causing tissue necrosis, inactivation of leukocytes and haemolysis of erythrocytes (8). Gas gangrene is one of the most life-threatening forms of necrotising infection due to the rapid destructive evolution and the massive involvement of muscle tissue. The onset of the disease is acute, and the earliest symptom a sense of increasing weight of the involved area soon followed by pain. Marked oedema and enzymatic necrosis of involved muscle cells are evident 1 to 3 days after injury. Gas caused by bacterial fermentation appears $\stackrel{c}{c}$ within the gangrenous tissues. As the infection progresses, the inflamed muscles become soft, blue-black, and semi-fluid as a $\bar{z}$ result of the proteolytic action of the released $\stackrel{\square}{\Omega}$ bacterial toxins. The hallmark of the disease $?$ is severe pain with subcutaneous crepitus, skin blistering and septic shock presenting late. Histologically there is severe $\underset{\vec{D}}{\vec{f}}$ myonecrosis, extensive haemolysis and marked vascular injury with thrombosis. The most common causative micro-organism is $\frac{\bar{s}}{\overrightarrow{2}}$ C. perfringens, which is present in more than $\mathbb{Q}$ $50 \%$ of cases(9). The mortality from 0 necrotising soft tissue infections ranges from $\overrightarrow{0}$ 4 to $85 \%$ (1). Early clinical diagnosis is of paramount importance. CT scan imaging is $\vec{\omega}$ able to delineate the extent of the disease and therefore direct the extent of debride- $\frac{9}{3}$ ment(10).

Clostridial myonecrosis following elective $\overrightarrow{\vec{A}}$ inguinal hernia repair is rare. Hitchcock et al $\dot{\omega}^{-}$ have reported two cases as part of their series $\frac{1}{\circ}$ of 133 patients(11). The origin of the infection in our patient was not identified. $\mathrm{He}$ was not at high risk of infection. The pure isolate of C. perfringens and normal findings $\frac{9}{0}$ at laparotomy do not support a bowel injury at hernia repair and sterilising procedures at the original hospital have been examined and exonerated.

In summary, clostridial myonecrosis is ao rare but devastating complication in surgery. Successful outcome is reliant on early recognition, radical debridement of necrotic tissue and multidisciplinary intensive care support.

\section{References}

1. Holley DT, McGrath PC, Sloan DA. Necrotizing fasciitis as a complication of percutaneous catheter drainage of an intra-abdominal abscess. Am Surg 1994; 60: 197-9.

2. Weinstein L, Barza MA. Gas gangrene. $N$ Engl $f($ Med 1973; 289: 1129-31.

3. Rouse TM, Malangoni MA, Schulte WJ. Necrotizing fasciitis: a preventable disaster. Surgery 1982; 92: 765-70.

4. Freischlag JA, Ajalat G, Busuttil RW. Treatment of necrotizing soft tissue infections. The need for a new approach. Am $\mathcal{F}$ Surg 1985; 149: 751-5.

5. McHenry CR, Piotrowski JJ, Petrinic $\mathrm{D}, \stackrel{D}{\supset}$ Malangoni MA. Determinants of mortality for을 necrotizing soft-tissue infections. Ann Surg 1995; 221: 558-63.

6. Urschel JD. Necrotizing soft tissue infections. N Postgrad Med f 1999; 75: 645-9.

7. Nichols RL, Smith JW. Anaerobes from a surgical $\omega$ perspective. Clin Infect Dis 1994; 18: S280-S286. ஜ

8. McDonel JL. Clostridium perfringens toxins (typeo A, B, C, D, E). Pharmacol Ther 1980; 10: 617-55.

9. Altemeier WA, Fullen WD. Prevention and treatment of gas gangrene. $\mathcal{F} A M A$ 1971; 217: 806- $\tau$ 13.

10. Wysoki MG, Santora TA, Shah RM, Friedman AC. Necrotizing fasciitis: CT characteristics. Radiology 1997; 203: 859-63.

11. Hitchcock CR, Demello FJ, Haglin JJ. Gangrene infection: new approaches to an old disease. Surg Clin North Am 1975; 55: 1403-10. 